\title{
ON THE GENERALISATION OF A FORMULA OF RAINVILLE
}

\section{JYOTI CHAUDHURI}

1. Introduction. Rainville [8] obtained a formula for $P_{n}(\operatorname{Cos} \alpha)$ where $P_{n}(x)$ is the Legendre polynomial. This was later generalised by Carlitz [5] who obtained a formula for $C_{n}^{\lambda+1 / 2}(\operatorname{Cos} \alpha)$, where $C_{n}^{\lambda}(x)$ is the Ultraspherical polynomial. Banerjee [1], Yadao [11] and Rangarajan [10] obtained similar formulae for the associated Legendre function $P_{n}^{m}(x)$.

The object of this short paper is to obtain a more general formula from which the above formulae follow as particular cases.

2. The generalised formula. Let $E(u)$ be the exponential function and let $G(u)$ possess a power series expansion (convergent or divergent)

$$
G(u)=\sum_{n=0}^{\infty} g_{n} u^{n}, \quad g_{n} \neq 0 .
$$

Define the sequence of polynomials $F_{n}(x)$ by the generating relation (essentially the one used in Example 21, p. 186, of Rainville [9])

$$
E(x t) G\left(\frac{1}{4} t^{2}\left(x^{2}-1\right)\right)=\sum_{n=0}^{\infty} \frac{F_{n}(x) t^{n}}{n !} .
$$

It follows, by multiplication of power series and equating coefficients of $t^{n}$, that

$$
F_{n}(x)=\sum_{k=0}^{\lfloor n / 2]} \frac{n ! g_{k} x^{n-2 k}\left(x^{2}-1\right)^{k}}{2^{2 k}(n-2 k) !} .
$$

The most useful special cases seem to occur when $G(u)$ is of hypergeometric form,

$$
G(u)={ }_{p} F_{q}\left[\begin{array}{l}
a_{1}, \cdots, a_{p} ; \\
b_{1}, \cdots, b_{q} ;
\end{array}\right]
$$

which leads to the polynomial set

(2.5) $\quad F_{n}(x)=x_{p+2}^{n} F_{q}\left[\begin{array}{c}-n / 2,-(n-1) / 2, a_{1}, \cdots, a_{p} ; \\ \frac{x^{2}-1}{x^{2}} \\ b_{1}, \cdots, b_{q} ;\end{array}\right]$.

Received by the editors July 27, 1965. 
Putting $t=v\left(y^{2}-1\right)^{1 / 2}$ in (2.2) we get

(2.6) $E\left[v x\left(y^{2}-1\right)^{1 / 2}\right] G\left[\frac{1}{4} v^{2}\left(x^{2}-1\right)\left(y^{2}-1\right)\right]=\sum_{n=0}^{\infty} \frac{v^{n}\left(y^{2}-1\right)^{n / 2}}{n !} F_{n}(x)$.

Interchanging $x$ and $y$,

(2.7) $E\left[v y\left(x^{2}-1\right)^{1 / 2}\right] G\left[\frac{1}{4} v^{2}\left(x^{2}-1\right)\left(y^{2}-1\right)\right]=\sum_{n=0}^{\infty} \frac{v^{n}\left(x^{2}-1\right)^{n / 2}}{n !} F_{n}(y)$.

Dividing (2.6) by (2.7) we have

$$
\begin{aligned}
& \sum_{n=0}^{\infty} \frac{v^{n}\left(y^{2}-1\right)^{n / 2}}{n !} F_{n}(x) \\
& \quad=E\left\{v\left(x\left(y^{2}-1\right)^{1 / 2}-y\left(x^{2}-1\right)^{1 / 2}\right)\right\} \sum_{n=0}^{\infty} \frac{v^{n}\left(x^{2}-1\right)^{n / 2}}{n !} F_{n}(y) .
\end{aligned}
$$

Now equating coefficients of $v^{n}$ from both sides, we obtain

$$
\text { (2.9) } F_{n}(x)=\left(\frac{1-x^{2}}{1-y^{2}}\right)^{n / 2} \sum_{k=0}^{n}\left(\begin{array}{l}
n \\
k
\end{array}\right)\left[\frac{x\left(1-y^{2}\right)^{1 / 2}-y\left(1-x^{2}\right)^{1 / 2}}{\left(1-x^{2}\right)^{1 / 2}}\right]^{n-k} F_{k}(y) \text {. }
$$

This can also be written as

$$
F_{n}(x)=\left(\frac{1-x^{2}}{1-y^{2}}\right)^{n / 2} \sum_{k=0}^{n}\left(\begin{array}{l}
n \\
k
\end{array}\right)\left[\frac{x\left(1-y^{2}\right)^{1 / 2}-y\left(1-x^{2}\right)^{1 / 2}}{\left(1-x^{2}\right)^{1 / 2}}\right]^{k} F_{n-k}(y)
$$

Here $F_{n}(x)$ is expressed as the sum of a series of $F_{n}(y)$.

3. Special cases of the above formula. Putting $x=\cos \alpha, y=\cos \beta$ in (2.9) we get,

$$
F_{n}(\cos \alpha)=\left(\frac{\sin \alpha}{\sin \beta}\right)^{n} \sum_{k=0}^{n}\left(\begin{array}{l}
n \\
k
\end{array}\right)\left[\frac{\sin (\beta-\alpha)}{\sin \alpha}\right]^{n-k} F_{k}(\cos \beta) .
$$

Putting $x=\sin \alpha, y=\cos \beta$ in (2.9) or changing $\alpha$ into $\pi / 2-\alpha$ in (3.1), (3.2) $F_{n}(\sin \alpha)=\left(\frac{\cos \alpha}{\sin \beta}\right)^{n} \sum_{k=0}^{n}(-1)^{n-k}\left(\begin{array}{l}n \\ k\end{array}\right)\left[\frac{\cos (\alpha+\beta)}{\cos \alpha}\right]^{n-k} F_{k}(\cos \beta)$. Putting $\beta=2 \alpha$ in (3.1),

$$
(2 \cos \alpha)^{n} F_{n}(\cos \alpha)=\sum_{k=0}^{n}\left(\begin{array}{l}
n \\
k
\end{array}\right) F_{k}(\cos 2 \alpha) .
$$

Putting $x=\sin \beta, y=-\cos \beta$ in (2.9) or changing $\alpha$ into $\pi / 2+\beta$ in (3.3) we get 


$$
\sin ^{n} \beta F_{n}(\sin \beta)=\sum_{k=0}^{n}(-1)^{k}\left(\begin{array}{l}
n \\
k
\end{array}\right) \cos ^{k} \beta F_{k}(\cos \beta) .
$$

Putting $y=-x$ in (2.9) we get

$$
F_{n}(x)=\sum_{k=0}^{n}(-1)^{k}\left(\begin{array}{l}
n \\
k
\end{array}\right)(2 x)^{n-k} F_{k}(x) .
$$

Putting $x=\cosh \alpha, y=\cosh \beta$ in (2.9) or changing $\alpha$ into $i \alpha$ and $\beta$ into $i \beta$ in (3.1), we get

(3.6) $F_{n}(\cosh \alpha)=\left(\frac{\sinh \alpha}{\sinh \beta}\right)^{n} \sum_{k=0}^{n}\left(\begin{array}{l}n \\ k\end{array}\right)\left[\frac{\sinh (\beta-\alpha)}{\sinh \alpha}\right]^{n-k} F_{k}(\cosh \beta)$.

Putting $x=\cos 2 \theta, y=\cos \theta$ in (2.9)

$$
F_{n}(\cos 2 \theta)=\sum_{k=0}^{n}(-1)^{n-k}\left(\begin{array}{l}
n \\
k
\end{array}\right)(2 \cos \theta)^{k} F_{k}(\cos \theta) .
$$

Putting $\cos \theta=x$ in (3.7)

$$
F_{n}\left(1-2 x^{2}\right)=\sum_{k=0}^{n}(-1)^{k}\left(\frac{n}{k}\right)(2 x)^{k} F_{k}(x) .
$$

Changing $x$ to $((1+x) / 2)^{1 / 2}$ and $y$ to $x$, we get from (2.9)

$$
2^{n / 2}(1+x)^{n / 2} F_{n}\left(\left(\frac{1+x}{2}\right)^{1 / 2}\right)=\sum_{k=0}^{n}\left(\begin{array}{l}
n \\
k
\end{array}\right) F_{k}(x) .
$$

Putting $(1-x t) / \rho$ for $x$ and $-x$ for $y$ in $(2.9)$, where $\rho=\left(1-2 x t+t^{2}\right)^{-1 / 2}$ we get

$$
\rho^{n} F_{n}\left(\frac{1-x t}{\rho}\right)=\sum_{k=0}^{n}(-1)^{k}\left(\begin{array}{l}
n \\
k
\end{array}\right) t^{k} F_{k}(x) .
$$

Putting $(x-t) / \rho$ for $x$ and $x$ for $y$ in (2.10), we have

$$
\rho^{n} F_{n}\left(\frac{x-t}{\rho}\right)=\sum_{k=0}^{n}(-1)^{k}\left(\begin{array}{l}
n \\
k
\end{array}\right) t^{k} F_{n-k}(x) .
$$

In this way by giving different values to $x$ and $y$, we can obtain various relations of similar types.

4. Particular cases. Putting $p=0, q=1$ and $b_{1}=1$ in (2.5), we see that $F_{n}(x)$ becomes the Legendre polynomial $P_{n}(x)$. Hence the formula (8) of Rainville [8] and its special cases become particular cases of (3.1), (3.3), (3.4), (3.5) and the result (3.9) above now re- 
duces to the result given by Bhonsle [2]. The results of Example 7 and Example 9, p. 184 of Rainville [9] follow easily from (3.4), (3.5), (3.8) and (3.10) respectively.

Putting $p=0, q=1, b_{1}=\lambda+\frac{1}{2}$ in (2.5) we find that $F_{n}(x)$ becomes

$$
\frac{n !}{(2 \lambda)_{n}} C_{n}^{\lambda}(x) \text {, }
$$

where $C_{n}^{\lambda}(x)$ is the Ultraspherical polynomial defined by $\left(1-2 x t+t^{2}\right)^{-\lambda}$ $=\sum C_{n}^{\lambda}(x) t^{n}$. The relation (2.9) then transforms into

$$
\begin{aligned}
C_{n}^{\lambda}(x) & =\left(\frac{1-x^{2}}{1-y^{2}}\right)^{n / 2} \\
\cdot & \sum_{k=0}^{n} \frac{(2 \lambda)_{n}}{(n-k) !(2 \lambda)_{k}}\left[\frac{x\left(1-y^{2}\right)^{1 / 2}-y\left(1-x^{2}\right)^{1 / 2}}{\left(1-x^{2}\right)^{1 / 2}}\right]^{n-k} C_{k}^{\lambda}(y) .
\end{aligned}
$$

With the substitution $y=2 x^{2}-1,(4.1)$ now reduces to the result (4.4) of Chatterjea [6]. The result given by Carlitz [5] is also a particular case of (4.1).

Denoting $\Phi_{n}(x)$ (as given by Rainville [8]) by the relation

$$
\Phi_{n}(x)=\left(1-x^{2}\right)^{n / 2} P_{n}\left(\frac{1}{\left(1-x^{2}\right)^{1 / 2}}\right)
$$

(2.9) reduces to

$$
y^{n} \Phi_{n}(x)=\sum_{k=0}^{n}\left(\begin{array}{l}
n \\
k
\end{array}\right)(y-x)^{n-k} x^{k} \Phi_{k}(y) .
$$

The results given by Chatterjea [6], [7] now follow easily from (4.3).

Putting $p=0, q=1, b_{1}=m+1$ in (2.5), we obtain

$$
F_{n}(x)=\left(x^{2}-1\right)^{-m / 2} \frac{2^{m} m ! n !}{(2 m) !(2 m+1)_{n}} P_{m+n}^{m}(x)
$$

and then (2.9) takes the form

$$
\begin{aligned}
P_{m+n}^{m}(x) & =\left(\frac{1-x^{2}}{1-y^{2}}\right)^{(m+n) / 2} \\
& \cdot \sum_{k=0}^{n}\left(\begin{array}{c}
2 m+n \\
k
\end{array}\right)\left(\frac{x\left(1-y^{2}\right)^{1 / 2}-y\left(1-x^{2}\right)^{1 / 2}}{\left(1-x^{2}\right)^{1 / 2}}\right)^{k} P_{m+n-k}^{m}(y) .
\end{aligned}
$$

The results (5), (6), (7) of Banerjee [1] and the result (2.3) of Rangarajan [10] now follow with proper substitutions from (4.4) as in $\$ 3$. 
From (3.10), putting $p=0, q=1, b_{1}=\alpha+1$, we get

$$
\rho^{n} \frac{n !}{(1+\alpha)_{n}} P_{n}^{(\alpha, \alpha)}\left(\frac{1-x t}{\rho}\right)=\sum_{k=0}^{n} \frac{(-n)_{k} P_{k}^{(\alpha, \alpha)}(x) t^{k}}{(1+\alpha)_{k}}
$$

which has been obtained by Brafman [4] by a different method. Similarly from (3.11) we obtain

$$
\rho^{n} C_{n}^{\nu}\left(\frac{x-t}{\rho}\right)=\sum_{k=0}^{n}(-1)^{k} \frac{t^{k}}{k !} \frac{\Gamma(n+2 \nu)}{\Gamma(n-k+2 \nu)} C_{n-k}^{\nu}(x) .
$$

Incidentally it may be mentioned that the result of Bloh [3]

$$
E(t z) I_{m}\left(t\left(z^{2}-1\right)^{1 / 2}\right)=\sum_{n=0}^{\infty} \frac{t^{m+n} P_{m+n}^{m}(z)}{(2 m+n) !}
$$

from which the results of Banerjee [1] were deduced, can be easily obtained from (2.2) with the substitutions $p=0, q=1, b_{1}=1+m$ and the definitions of $P_{m+n}^{m}(x)$ and $I_{m}(x)$.

In conclusion, I wish to express my gratitude to Professor B. N. Mukherjee for his kind help and guidance in preparation of this paper.

\section{REFERENCES}

1. D. P. Banerjee, On some results involving associated Legendre's functions, Boll. Un. Mat. Ital. (3) 16 (1961), 218-220.

2. B. R. Bhonsle, On a series of Rainville involving Legendre polynomials, Proc. Amer. Math. Soc. 8 (1957), 10-14.

3. E. L. Bloh, On an expansion of Bessel functions in a series of Legendre functions, Prikl. Mat. Meh. 18 (1954), 745-748.

4. F. Brafman, Generating functions of Jacobi and related polynomials, Proc. Amer. Math. Soc. 2 (1951), 942-949.

5. L. Carlitz, Note on a formula of Rainville, Bull. Calcutta Math. Soc. 51 (1959), 132-133.

6. S. K. Chatterjea, On a series of Carlitz involving ultraspherical polynomials, Rend. Sem. Mat. Univ. Padova 31 (1961), 294-300.

7. - Note on a formula of Carlitz, Rend. Sem. Mat. Univ. Padova 31 (1961), $243-248$.

8. E. D. Rainville, Notes on Legendre polynomials, Bull. Amer. Math. Soc. 51 (1945), 268-271.

9. - Special functions, Macmillan, New York, 1960.

10. S. K. Rangarajan, On a new formula for $P_{m+n}^{m}(\cos \alpha)$, Quart. J. Math. 15 (1964), 31-34. 30.

11. G. M. Yadao, $A$ new formula for $P_{m+n}^{m}(\cos \alpha)$, Quart. J. Math. 13 (1962), 29-

JADAVPUR UNIVERSITY

Calcutta, India 